\title{
Broad Specificity of Amino Acid Chemoreceptor CtaA of Pseudomonas fluorescens Is Afforded by Plasticity of Its Amphipathic Ligand-Binding Pocket
}

\author{
Abu I. M. S. Ud-Din, ${ }^{1}$ Mohammad F. Khan, ${ }^{2}$ and Anna Roujeinikova ${ }^{1,2,+}$ \\ ${ }^{1}$ Infection and Immunity Program, Monash Biomedicine Discovery Institute, Australia, Department of Microbiology, Monash \\ University, Clayton, Victoria 3800, Australia \\ ${ }^{2}$ Department of Biochemistry and Molecular Biology, Monash University, Clayton, Victoria 3800, Australia
}

Accepted 24 December 2019.

\begin{abstract}
Motile bacteria follow gradients of nutrients or other environmental cues. Many bacterial chemoreceptors that sense exogenous amino acids contain a double Cache (dCache; calcium channels and chemotaxis receptors) ligand-binding domain (LBD). A growing number of studies suggest that broad-specificity dCache-type receptors that sense more than one amino acid are common. Here, we present an investigation into the mechanism by which the dCache LBD of the chemoreceptor $\mathrm{CtaA}$ from a plant growth-promoting rhizobacterium, Pseudomonas fluorescens, recognizes several chemically distinct amino acids. We established that amino acids that signal by directly binding to the CtaA LBD include ones with aliphatic (L-alanine, $\mathrm{L}$-proline, $\mathrm{L}$-leucine, $\mathrm{L}$-isoleucine, $\mathrm{L}$-valine), small polar (L-serine), and large charged (L-arginine) side chains. We determined the structure of CtaA LBD in complex with different amino acids, revealing that its ability to recognize a range of structurally and chemically distinct amino acids is afforded by its easily accessible plastic pocket, which can expand or contract according to the size of the ligand side chain. The amphipathic character of the pocket enables promiscuous interactions with both polar and nonpolar amino acids. The results not only clarify the means by which various amino acids are recognized by $\mathrm{CtaA}$ but also reveal that a conserved mobile lid over the ligand-binding pocket adopts the same conformation in all complexes, consistent with this being an important and invariant part of the signaling mechanism.
\end{abstract}

Keywords: chemotaxis, crystal structure, methyl-accepting protein, sensing domain

Pseudomonas fluorescens is a gram-negative plant growthpromoting rhizobacterium (PGPR) that is widely distributed in nature, often found on plant roots, in soil, and in water (Compant et al. 2005; Glick 2012; Lugtenberg and Kamilova

${ }^{\dagger}$ Corresponding author: A. Roujeinikova; anna.roujeinikova@monash.edu

Funding: This work was funded by Australian Research Council grant DP180101807.

*The $\boldsymbol{e}$-Xtra logo stands for "electronic extra" and indicates that supplementary materials are published online.

The author(s) declare no conflict of interest.

(c) 2020 The American Phytopathological Society
2009; Vacheron et al. 2013; van Loon et al. 1998; Vejan et al. 2016). Different PGPR species stimulate plant growth via a wide range of direct and indirect mechanisms. They act as a biofertilizer, i.e., provide nutrients to plants by solubilizing phosphate, reducing atmospheric nitrogen to ammonia, and producing siderophores to increase soil iron availability. They also produce phytohormones, including indole-3-acetic acid, gibberellins, and cytokinins. In addition, root colonization with this bacterium has been shown to induce systemic resistance against phytopathogens. Furthermore, PGPR enhance plant growth by producing antifungal and antibacterial compounds and help plants tolerate biotic and abiotic stress by expressing 1-aminocyclopropane-1-carboxylate deaminase (Compant et al. 2005; Glick 2012; Vacheron et al. 2013; van Loon et al. 1998; Vejan et al. 2016).

Chemotaxis toward root exudate components, such as organic acids and amino acids, plays a major role in root colonization by Pseudomonas fluorescens (de Weert et al. 2002; Oku et al. 2012, 2014). Chemotaxis is mediated by methylaccepting chemotaxis proteins (MCPs), bacterial receptors that sense chemoeffector gradients in the environment (Ud-Din and Roujeinikova 2017). A typical MCP, also known as a chemoreceptor, consists of an extracytoplasmic ligand-binding domain (LBD) flanked by two transmembrane (TM) helices, followed by a HAMP (histidine kinase, adenyl cyclase, MCP, and phosphatase) domain and a cytoplasmic signaling domain. The signaling domain interacts with downstream regulatory proteins to control the rotation of flagella (Briegel et al. 2009; Wadhams and Armitage 2004).

While the signaling domains are highly conserved, LBDs are extremely diverse in terms of their primary sequence and structure (Krell et al. 2011). They are classified into two major clusters based on their size: cluster I (average length 156 amino acids) and cluster II (average length 262 amino acids) (Lacal et al. 2010). Cluster I includes the PAS (per-Arnt-sim), the single Cache (calcium channels and chemotaxis receptors), and the 4HB (4-helix bundle) domains (Matilla and Krell 2017). Within cluster II, two structural families have been identified to date, i.e., the helical bimodular (HBM) domains (Ortega and Krell 2014) and the double Cache (dCache) domains (Glekas et al. 2012; Liu et al. 2015; Machuca et al. 2016, 2017; Nishiyama et al. 2016; Takahashi et al. 2019). Together, the single Cache and the dCache domains represent the most abundant type of extracytoplasmic sensing domains in prokaryotes (Upadhyay et al. 2016).

The P. fluorescens Pf0-1 genome harbors genes for 37 MCPs (Oku et al. 2012), none of which have been structurally 
characterized to date. Microbiological studies identified the products of genes Pf101_4431, Pf101_0124, and Pf101_0354 as chemoreceptors for amino acids, and they have been termed chemotactic transducers of amino acids $\mathrm{CtaA}, \mathrm{CtaB}$, and $\mathrm{CtaC}$. The CtaA LBD comprising amino acid residues 34 to 277 (UdDin and Roujeinikova 2016), shares 65\% amino acid sequence identity with the LBD of PctA from Pseudomonas aeruginosa PAO1, a chemoreceptor that senses (directly or indirectly) 18 natural amino acids (Oku et al. 2012; Rico-Jiménez et al. 2013; Taguchi et al. 1997). Similar to PctA, P. fluorescens CtaA has broad specificity, mediating taxis toward 18 different amino acids (Oku et al. 2012).

A search against the Pfam database (v.29.0) (Finn et al. 2014) using the primary sequence of CtaA LBD indicated that, like PctA LBD, CtaA LBD belongs to the dCache family (Pfam accession number PF02743). Previous structural studies revealed that receptors with a dCache LBD that recognizes amino acids directly, contain conserved consensus motif DXXX(R/K)CWYXXA (Liu et al. 2015). This motif is present in $P$. fluorescens CtaA, suggesting that this receptor senses at least some of its amino acid ligands via direct binding. In addition to $P$. fluorescens and $P$. aeruginosa, broad-specificity dCache-type chemoreceptors for multiple amino acids, containing this conserved consensus motif, have been found in Sinorhizobium meliloti (McpU [Webb et al. 2017]) Bacillus subtilis (McpC [Glekas et al. 2012]), and Vibrio cholerae (Mlp24 [Takahashi et al. 2019] and Mlp37 [Nishiyama et al. 2016]), suggesting that such promiscuous amino acid receptors are widespread.

Previous analysis of the crystal structures of Mlp24 LBD in complex with different amino acids revealed that ligands with a short side chain bind to a relatively small pocket in the membrane-distal subdomain of the receptor in a very similar mode (Takahashi et al. 2019). Unfortunately, no crystal structure of LBD of Mlp24 or any other homologous receptor in complex with an amino acid with a large aliphatic side chain (e.g., Mlp24/methionine [Takahashi et al. 2019]) is available, leaving open the important question of how the same receptor can recognize both polar and apolar amino acids. Our recent discovery of a dCache-type Helicobacter pylori chemoreceptor, $\mathrm{TlpC}$, that directly senses lactate via its membrane-proximal rather than membrane-distal subdomain (Machuca et al. 2017), raised the possibility that promiscuous receptors, such as CtaA, may use both subdomains for signal recognition. Cumulatively, the data available so far emphasized the necessity for further biophysical and crystallographic studies of broad-spectrum chemoreceptors in complexes with different amino acids.

Here, we present an analysis of recombinant CtaA LBD (residues 34 to 277) from $P$. fluorescens, which shows that seven of the 18 natural amino acids that signal through CtaA bind to it directly. Determination of the crystal structures of CtaA LBD in complex with the six tightest binders allowed identification of the residues important for ligand recognition and the underlying structural basis for the broad specificity of the receptor. This report provides a detailed structural account of the plasticity of the ligand-binding pocket that enables a dCache MCP receptor to interact with many chemically distinct amino acids.

\section{RESULTS}

Amino acid binding profile of CtaA LBD.

To determine which of the 18 amino acids that signal via $P$. fluorescens $\mathrm{CtaA}$ bind to its LBD directly, isothermal titration calorimetry (ITC) experiments were carried out with all 20 natural L-amino acids. Seven amino acids (L-valine, L-alanine, L-serine, L-leucine, L-proline, L-isoleucine, and L-arginine) showed binding to CtaA LBD, with apparent affinities ranging from 4.7 to $446.8 \mu \mathrm{M}$ (Table 1; Fig. 1). The binding was exothermic for L-alanine, L-serine, and L-proline but endothermic for L-valine, L-leucine, L-isoleucine, and L-arginine. CtaA LBD showed the highest affinities for L-valine (dissociation constant $\left.\left(K_{\mathrm{d}}\right)=4.7 \mu \mathrm{M}\right)$ and L-alanine $\left(K_{\mathrm{d}}=5.2 \mu \mathrm{M}\right)$. The affinity for L-arginine was significantly lower $\left(K_{\mathrm{d}}=446.8 \mu \mathrm{M}\right)$, compared with the other six amino acids. The calorimetric measurements also confirmed that the two amino acids not sensed by CtaA (L-glutamate and L-glutamine) do not bind to its LBD.

\section{Overall fold of CtaA LBD.}

Crystallization of CtaA LBD with or without various amino acid ligands yielded two different crystal forms. Crystals grown in the presence of L-alanine, L-valine, L-serine, L-leucine, or L-proline or in the absence of added ligand belonged to space group $I 2_{1} 2_{1} 2_{1}$, with one protein subunit in the asymmetric unit. These crystals are hereafter referred to as form A. Cocrystallization with $\mathrm{L}$-isoleucine under similar conditions yielded form $\mathrm{B}$ crystals that had $C 2$ symmetry and contained two subunits in the asymmetric unit.

The crystal structure of the recombinant $P$. fluorescens CtaALBD cocrystallized with L-alanine was determined at $2.0 \AA$ resolution, using the molecular replacement method. As illustrated in Figure 2, CtaA LBD adopts a dCache fold consisting of two PAS-like subdomains, termed membrane-distal (residues 61 to 175) and membrane-proximal (residues 34 to 60, 176 to 277). The long N-terminal helix $\alpha 1$ (residues 34 to 77) forms part of both subdomains.

The core of the membrane-distal subdomain is an antiparallel $\beta$-sheet consisting of five $\beta$-strands arranged in the topological order $\beta 2 \downarrow \beta 1 \uparrow \beta 5 \downarrow \beta 4 \uparrow \beta 3 \downarrow$ (Fig. 2B). This $\beta$-sheet is sandwiched between an antiparallel helix bundle (helices $\alpha 2, \alpha 2$ ', and the C-terminal half of the $\alpha 1$-helix) on one side and helix $\alpha 3$ on the other side. The core of the membrane-proximal subdomain is also a five-stranded antiparallel $\beta$-sheet with its strands arranged in the order $\beta 7 \downarrow \beta 6 \uparrow \beta 10 \downarrow \beta 9 \uparrow \beta 8 \downarrow$. Helix $\alpha 4$ and the $\mathrm{N}$-terminal half of helix $\alpha 1$ are packed against one face of this $\beta$-sheet, while helix $\alpha 5$ is packed against the other. In the fulllength receptor, the $\mathrm{N}$ terminus of helix $\alpha 1$ would be linked to the transmembrane helix TM1 and the C terminus of helix $\alpha 6$ to the helix TM2. The structural folds of the membrane-distal and membrane-proximal subdomains are very similar and can be superimposed over $80 \mathrm{C}_{\alpha}$ atoms with a root-mean-square

Table 1. Thermodynamic parameters of binding of L-amino acids to CtaA ligand-binding domain derived from isothermal titration calorimetry measurements ${ }^{\mathrm{a}}$

\begin{tabular}{|c|c|c|c|}
\hline Ligand & $K_{\mathrm{d}}(\mu \mathrm{M})$ & Enthalpy, $\Delta H\left(\right.$ cal $\mathrm{mol}^{-1}$ ) & Entropy, $\Delta S\left(\right.$ cal $\left.\mathrm{mol}^{-1} \mathrm{~K}^{-1}\right)$ \\
\hline L-valine & $4.7 \pm 1.2$ & $1,703 \pm 69.3$ & $30.1 \pm 0.7$ \\
\hline L-alanine & $5.2 \pm 0.3$ & $-1,906 \pm 53.7$ & $17.8 \pm 1.4$ \\
\hline L-serine & $9.5 \pm 1.1$ & $-1,853 \pm 43.1$ & $16.8 \pm 0.5$ \\
\hline L-leucine & $11.9 \pm 1.8$ & $1,896 \pm 126.6$ & $28.9 \pm 0.1$ \\
\hline L-proline & $13.5 \pm 0.3$ & $-2,926 \pm 31.8$ & $12.5 \pm 0.2$ \\
\hline L-isoleucine & $27.4 \pm 0.9$ & $2,091 \pm 93.7$ & $27.9 \pm 1.6$ \\
\hline L-arginine & $446.8 \pm 17.6$ & $1,521 \pm 58.7$ & $20.4 \pm 0.3$ \\
\hline
\end{tabular}

${ }^{\mathrm{a}}$ Data are means and standard deviations from three experiments; cal $\mathrm{mol}^{-1} \mathrm{~K}^{-1}=$ calories per mole per kelvin. 
deviation (RMSD) of $2.4 \AA$, despite the very low (17\%) overall amino acid sequence identity between them. There are extensive interactions between the two subdomains, with approximately $1,510 \AA^{2}$ of the total accessible surface area buried at their interface.
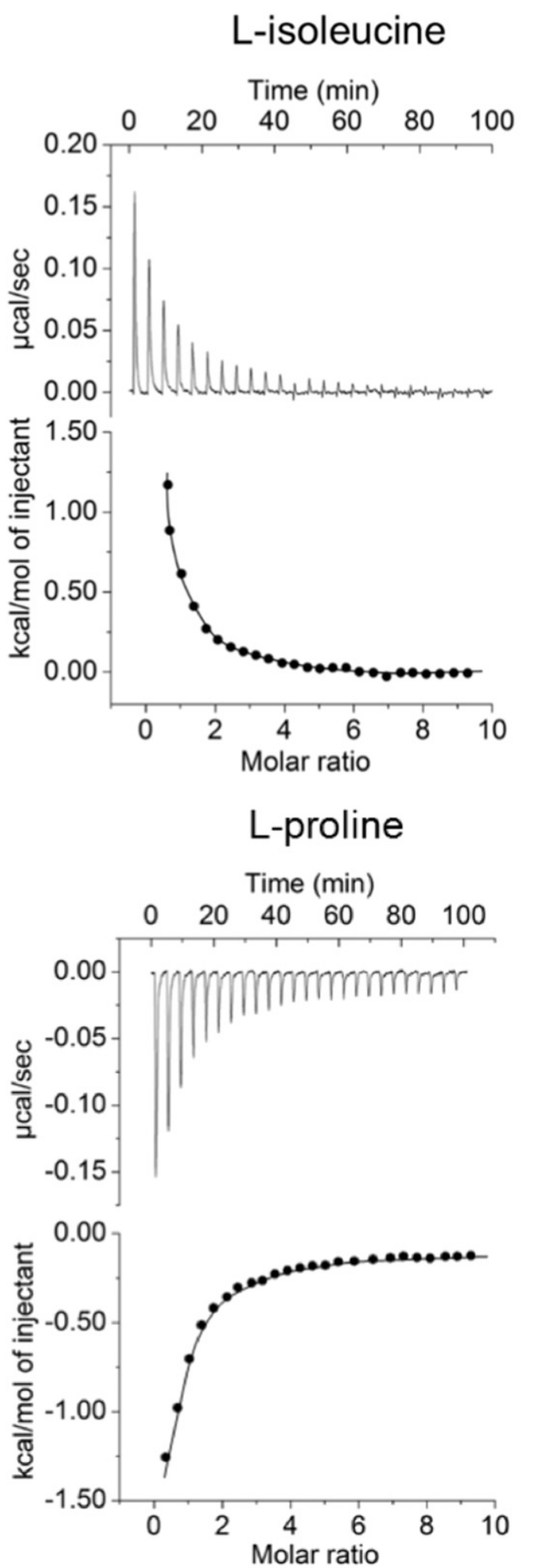

Comparison with other members of the dCache structural family.

Comparison of CtaA LBD with structures available in the Protein Data Bank (PDB) (Berman et al. 2007), using the PDBeFold server (Krissinel and Henrick 2004), revealed
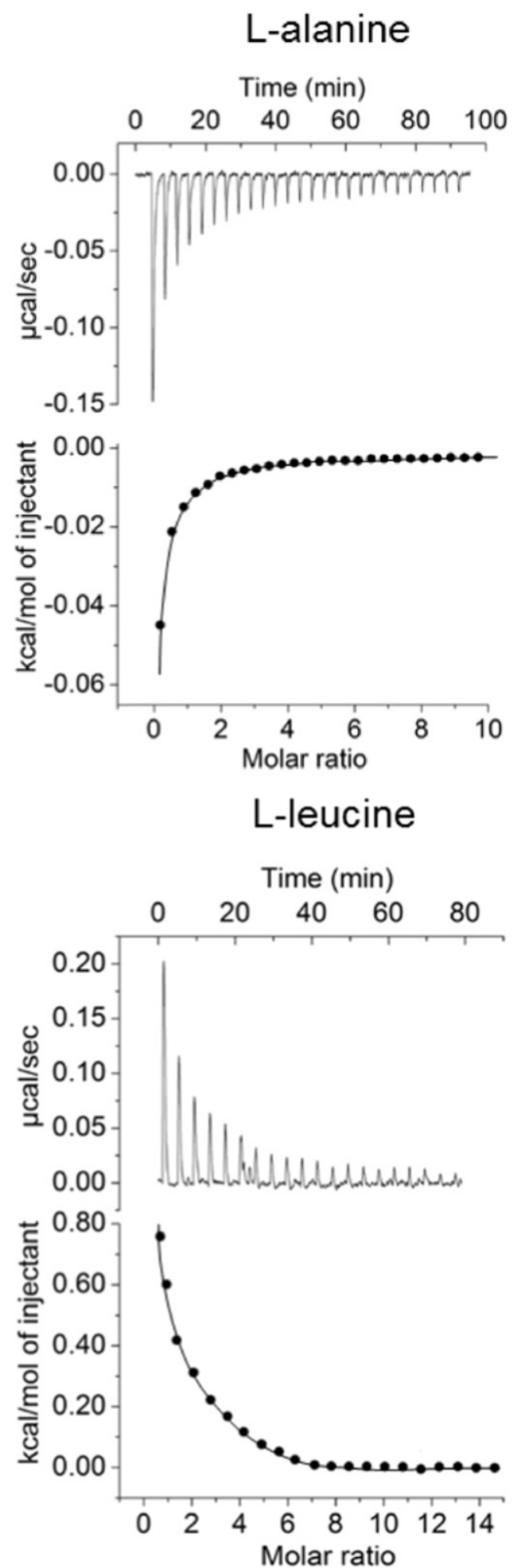

Fig. 1. Representative isothermal titration calorimetry data for Pseudomonas fluorescens CtaA ligand-binding domain (LBD) and different amino acids. Each peak in the top panels corresponds to the injection of $10 \mu \mathrm{l}$ of the amino acid solution into a $1.45-\mathrm{ml}$ reaction cell containing protein at a concentration of $10 \mu \mathrm{M}$. The cumulative heat of the reaction is shown in the bottom panels as a function of the amino acid/protein molar ratio. The solid line represents the least-squares fit of the experimental data to a single-site binding model. 
significant similarities to other dCache LBDs of transmembrane receptors. The top five hits were $V$. cholerae Mlp37 (VcMlp37, PDB ID 5AVF [Nishiyama et al. 2016], RMSD 1.8 $\AA$ for the superimposition over $244 \mathrm{C}_{\alpha}$ atoms), Methanosarcina mazei family 1 histidine kinase Z3 $\left(\mathrm{MmHK}_{1} \mathrm{Z} 3\right.$, PDB ID $3 \mathrm{LIB}$ [Zhang and Hendrickson 2010], RMSD $2.1 \AA$ for the superimposition over $271 \mathrm{C}_{\alpha}$ atoms), M. mazei family 1 histidine kinase Z2 ( $\mathrm{MmHK}_{1} \mathrm{Z} 2$, PDB ID 3LI8 [Zhang and Hendrickson 2010], RMSD $2.3 \AA$ for the superimposition over $263 \mathrm{C}_{\alpha}$ atoms), Campylobacter jejuni chemoreceptor Tlp1 (CjTlp1, PDB ID 4WY9 [Machuca et al. 2016], RMSD $2.3 \AA$ for the superimposition over $285 \mathrm{C}_{\alpha}$ atoms), and $C$. jejuni chemoreceptor Tlp3 (CjTlp3, PDB ID 4XMR [Liu et al. 2015], RMSD $2.5 \AA$ for superimposition over $253 \mathrm{C}_{\alpha}$ atoms) (Fig. 3A). Analysis of the superimposed structures revealed that structural similarity extends over the entire fold and includes most secondary structure elements.

Previously characterized dCache LBDs that recognize amino acids directly form dimers in the crystal, and the dimerization mode appears to be conserved (Liu et al. 2015; Nishiyama et al. 2016; Takahashi et al. 2019). In a typical dimer, exemplified in Figure 3B by CjTlp3 LBD (Liu et al. 2015), the two long stalk helices are nearly parallel to each other. Similar dimers have been observed in the crystals of dCache LBDs of non-MCP receptors, such as histidine kinases $\mathrm{MmHK}_{1} \mathrm{Z3}$ (Zhang and Hendrickson 2010) and Sinorhizobium meliloti DctB (Zhou et al. 2008) (Fig. 3B). However, dimers in which the two halves associate in the crystal lattice in a related but different manner have also been reported. For example, in the dimers observed in the crystal of VcMlp37 LBD (Nishiyama et al. 2016) and in some of the crystals of VcMlp24 LBD (Takahashi et al. 2019), the two stalk helices are positioned with their N-terminal ends apart (Fig. 3B). This fact prompted us to interrogate the quaternary structure of CtaA LBD in the crystal, using the PDBePISA (protein interfaces, surfaces, and assemblies) server (Krissinel and Henrick 2007) at the European Bioinformatics Institute. The analysis showed that form A and form B crystals of CtaALBD are also composed of dimers that resemble the ones seen in the crystals of other dCache LBDs. In both forms, the mode of dimerization (with the N-terminal ends of the two stalk helices apart) is more like that of VcMlp37 LBD than CjTlp3LBD (Fig. 3B). However, the association appears to be unstable (small and negative free energy of dissociation $\Delta \mathrm{G}^{\text {diss }}=$ $-1.2 \mathrm{kcal} / \mathrm{mol}$ ), which is in agreement with our previous observation that CtaA-LBD is a monomer in solution (Ud-Din and Roujeinikova 2016). Therefore, the biological relevance of the observed crystallographic dimer cannot be postulated.

\section{Analysis of binding modes of amino acids.}

Analysis of the molecular surface topology of CtaA LBD, using the Depth server (Tan et al. 2013), suggested that both the membrane-distal and membrane-proximal subdomains contain putative ligand-binding pockets. To pinpoint the binding sites for different amino acids and compare their binding modes, we determined and analyzed the crystal structures of CtaA LBD in complex with the six tightest binders (L-alanine, L-valine, L-serine, L-proline, L-leucine, and L-isoleucine). The coordinates of the protein molecules from the six complexes could be superimposed with an average pairwise C $\alpha$ RMSD of $0.5 \AA$, showing no significant differences. We have therefore focused our analysis on the similarities and differences in the relative positions of the respective amino acid ligands and protein side chains in the binding pocket.

Analysis of the electron density map calculated for the cocrystal with L-alanine revealed unambiguous electron density for the ligand in the pocket in the membrane-distal subdomain
(Fig. 4A), which is the common binding site for amino acidcomplexed structures of dCache LBDs (Liu et al. 2015; Nishiyama et al. 2016; Takahashi et al. 2019). This pocket is formed by one face of the central $\beta$-sheet, helix $\alpha 3$, and loops $\beta 2 \alpha 3$ and $\beta 3 \beta 4$, and is lined by residues Y101, F109, I111, M117, F121, R126, W128, Y144, D146, A147, A148, I153, and D173. The amino group of L-alanine forms hydrogen bonds with $\mathrm{Y}_{1440}{ }^{\eta}, \mathrm{D} 146 \mathrm{O}^{\delta 1}$, and $\mathrm{D} 173 \mathrm{O}^{\delta 2}$, while its carboxyl oxygen atoms form hydrogen bonds with $\mathrm{R} 126 \mathrm{~N}^{\varepsilon}, \mathrm{R} 126 \mathrm{~N}^{\eta 2}, \mathrm{~W} 128 \mathrm{~N}^{\varepsilon 1}$, and with the main-chain amino group of A147 (Fig. 4A; Table 2). L-alanine is further stabilized by van der Waals contacts of its side-chain methyl group with Y101, F109, I111, and M117.

Comparison of the structures of CtaA LBD in complex with L-alanine, L-valine, L-serine (Fig. 4A, B, and C, respectively), L-leucine, L-isoleucine, and L-proline (Fig. 5A, B, and C,
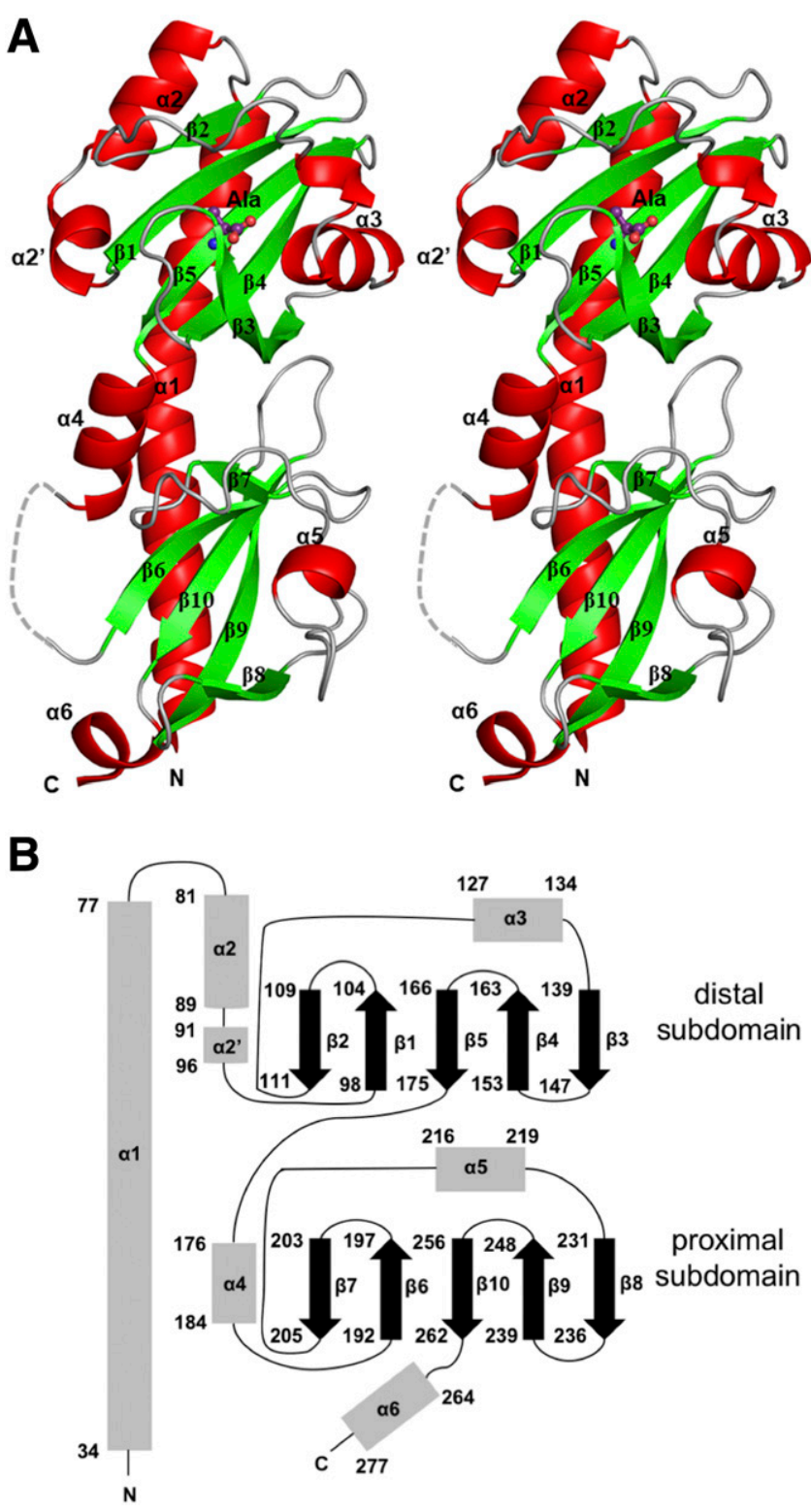

Fig. 2. Overall fold of Pseudomonas fluorescens CtaA ligand-binding domain (LBD). A, A stereo representation of the structure of the CtaA LBD monomer. $\alpha$-Helices and $\beta$-strands are represented by red coils and green arrows. The alanine molecule is shown in ball-and-stick representation, with carbon atoms colored purple, to indicate the location of the ligandbinding site. B, The topology of the secondary structure elements of CtaA LBD. 
respectively) shows that the binding modes of all six amino acids are very similar. The hydrogen bonds between the protein and the amino and carboxyl groups of the ligand, described above for L-alanine, are present in all other complexes, except for the hydrogen bond to $\mathrm{Y}_{144 \mathrm{O}^{\eta}}$, which is not present in the complex with L-proline. The aliphatic side chains of L-alanine, L-valine, L-leucine, and L-isoleucine in their respective complexes with CtaA LBD are in a largely hydrophobic environment and are stabilized by multiple van der Waals contacts with apolar side chains of the protein (Table 2; Figs. 4 and 5). Yet, the same pocket can also accommodate the small, polar side chain of L-serine by allowing a hydrogen bond between its $\mathrm{O}^{\gamma}$ atom and ${\mathrm{D} 146 \mathrm{O}^{\delta 2}}^{\delta}$ (Fig. 4C). Table 2 details the stabilizing interactions between $\mathrm{CtaA}$ LBD and its ligands in the respective complexes.

We note that according to our calorimetry data, amino acids with branched aliphatic side chains (L-valine, L-leucine and Lisoleucine) bind to CtaA LBD endothermically, in contrast to Lalanine, L-serine, and L-proline, which bind exothermically (Table 1). This indicates that binding of ligands with branched aliphatic side chains to $\mathrm{CtaA}$ is entropically driven, which suggests that hydrophobic effects (such as the release of bound solvent as the apolar side chain of the ligand binds to the apolar side chains of the protein molecule) make a key contribution to binding of these ligands. The protein-ligand interactions observed in the crystals structures of the respective complexes are therefore consistent with the calorimetry data.
The ligand-binding (membrane-distal) subdomains of dCache LBDs of CjTlp3 and VcMlp24, which also sense amino acids by directly binding to them, were previously reported to undergo a significant conformational change upon amino acid binding. Ligand binding to these proteins triggers a closure of the 'lid' - the loop corresponding to loop $\beta 3 \beta 4$ in CtaA LBD-over the ligand-binding pocket (Liu et al. 2015; Takahashi et al. 2019). Comparisons of the crystal structures of the CtaA LBD/amino acid complexes with those of the open (free) and closed (isoleucine-bound) forms of CjTlp3 LBD (Liu et al. 2015) (Supplementary Fig. S1) showed that the membrane-distal subdomain of the ligandbound CtaA LBD adopts a closed conformation, which is consistent with the behavior of LBDs of CjTlp3 and VcMlp24.

Crystallization of CtaA LBD with no amino acid added to the purification or crystallization buffers yielded form A crystals with a small molecule seen bound in the ligandbinding pocket and the membrane-distal subdomain adopting a closed conformation. The shape of the electron density for the ligand was consistent with L-alanine, and the crystals were isomorphous to the form A cocrystals with L-alanine. The protein molecules for the crystals grown with or without Lalanine could be superimposed based on the overlap of $225 \mathrm{C}_{\alpha}$ atoms with an RMSD of $0.15 \AA$, indicating that, within the limit of the experimental error in the coordinates $(0.20 \AA$ for the cocrystal with L-alanine), their structures were essentially
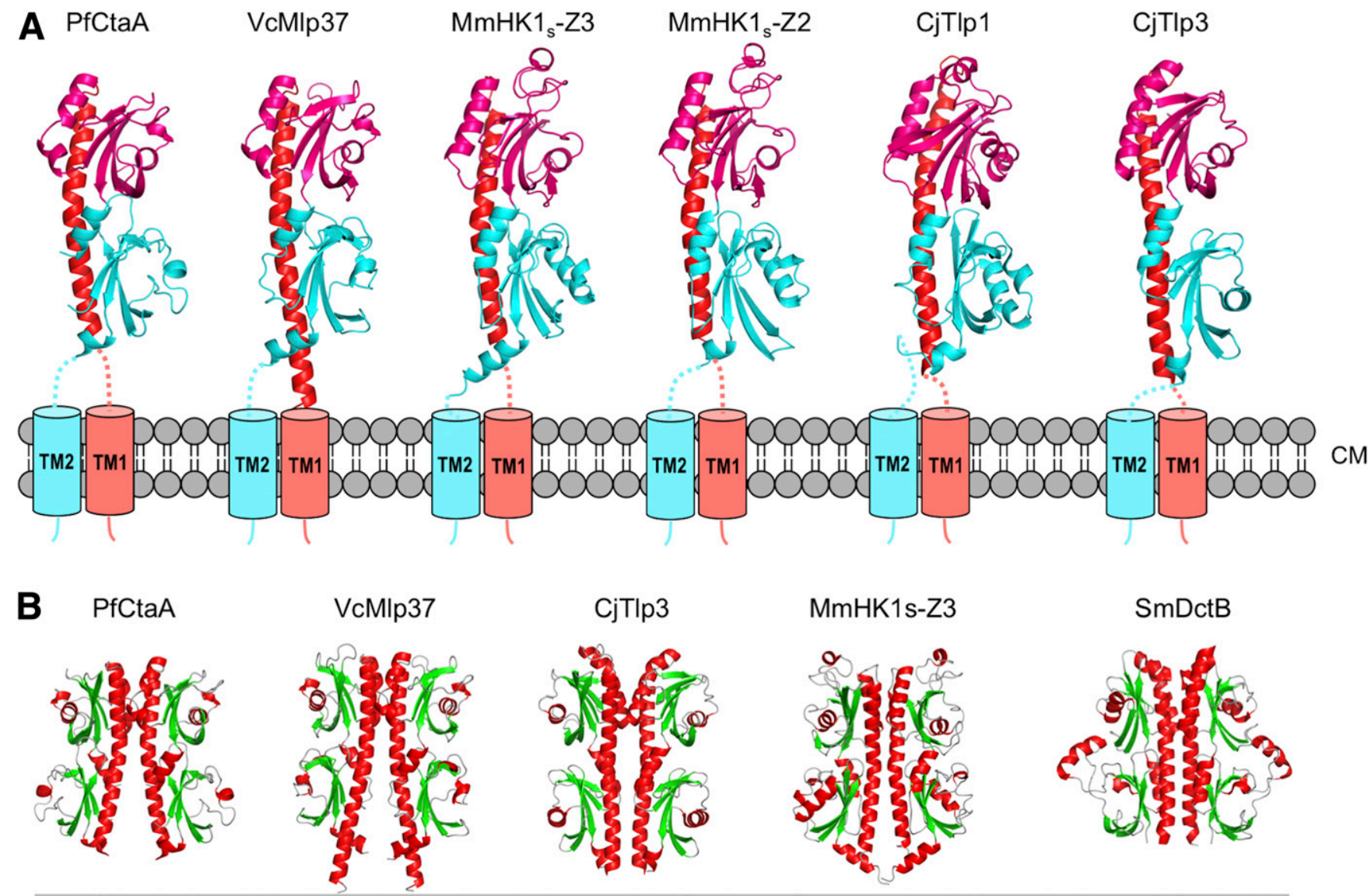

cytoplasmic membrane

Fig. 3. Comparison of Pseudomonas fluorescens CtaA ligand-binding domain (LBD) to other members of double Cache structural family. A, A side-by-side view of single subunits of extracytoplasmic LBDs of P. fluorescens CtaA, Vibrio cholerae Mlp37, Methanosarcina mazei family 1 histidine kinases Z2 and Z3, Campylobacter jejuni Tlp1, and C. jejuni Tlp3. B, LBD dimers of P. fluorescens CtaA, V. cholerae Mlp37, C. jejuni Tlp3, M. mazei family 1 histidine kinase Z3, and $S$. meliloti DetB as found in the crystal structures. 
identical. When the model was refined with the ligand modeled as L-alanine, its binding mode was found to be highly similar to that seen in the cocrystals with L-alanine. Presumably, L-alanine was captured by the protein from the Escherichia coli cell lysate during expression and purification. Thus, no structure of unliganded CtaA LBD is available. Similarly, cocrystallization of CtaA LBD with $5 \mathrm{mM}$ L-arginine, the weakest-binding ligand, yielded form A crystals with L-alanine bound in the ligand-binding pocket (data not shown), which presumably copurified with the protein and could not be displaced by Larginine due to an approximately 100-fold difference in their binding affinities.
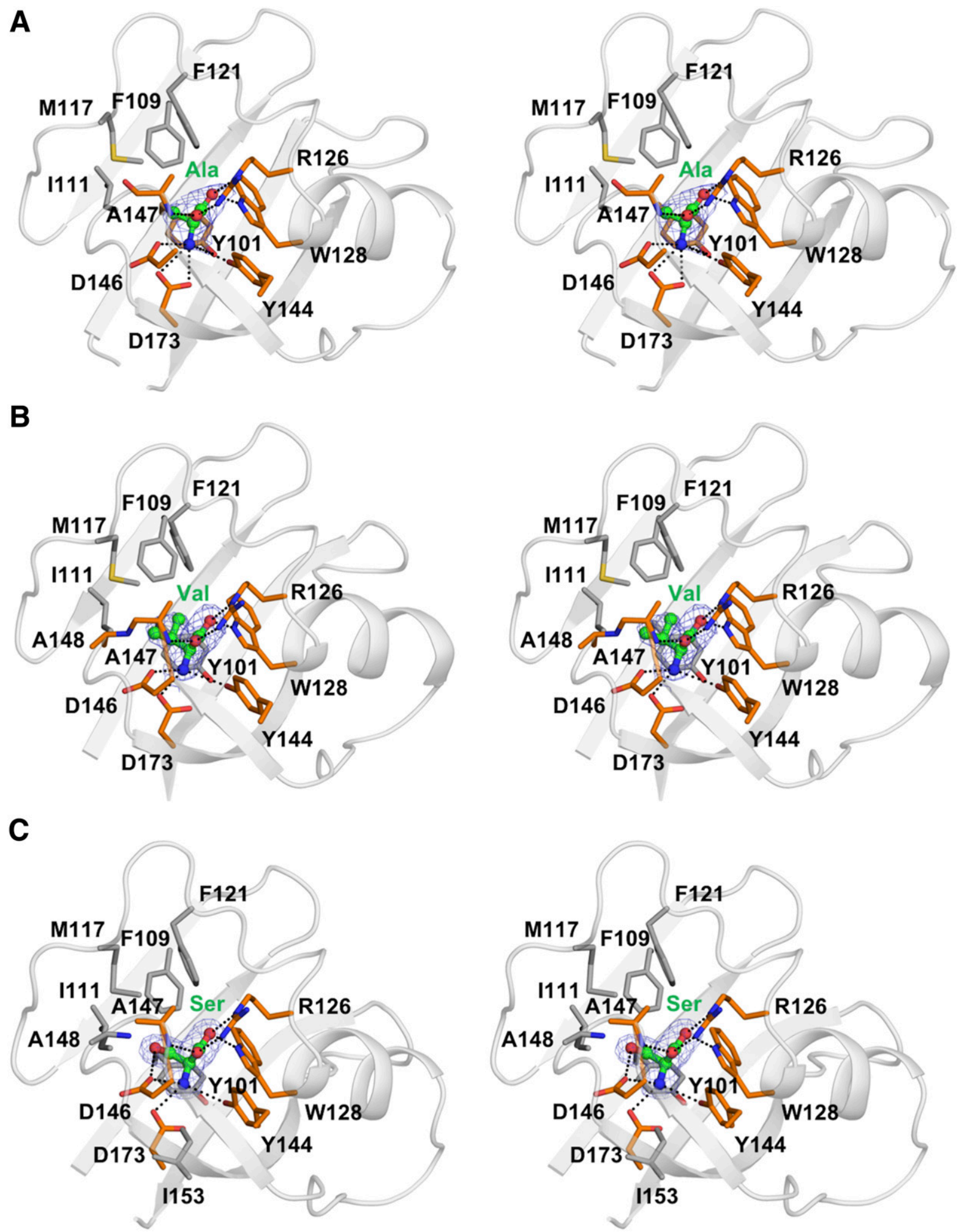

Fig. 4. Stereo diagrams showing simulated annealing omit electron density for amino acids bound to the membrane-distal subdomain of Pseudomonas fluorescens $\mathrm{CtaA}$ in respective complexes with $\mathbf{A}, \mathrm{L}$-alanine, $\mathbf{B}, \mathrm{L}$-valine, and $\mathbf{C}, \mathrm{L}$-serine. The ligands are shown in all-atom ball-and-stick representation with $\mathrm{C}$ atoms colored green. The map was contoured at the $1.0 \sigma$ level. The residues that form hydrogen bonds and van der Waals contacts with the ligand are shown as sticks with carbons colored orange and gray, respectively. 


\section{Conformational plasticity}

of the amphipathic ligand-binding pocket.

To obtain a structural rationale for how chemically distinct amino acids can bind to the same pocket in CtaA LBD in a very similar orientation, we have analyzed the topology of the ligand-binding pocket in the respective complexes. This analysis revealed several important feautures. First, as showin in Figure $6 \mathrm{~A}$ to $\mathrm{F}$, the pocket is connected to the bulk solvent via a short tunnel. This tunnel, filled with ordered water molecules, likely serves as a ligand entry/exit pathway. Even in the closed form of the protein, access to the ligand-binding pocket through this tunnel is only partially hindered by the loop $\beta 3 \beta 4$, suggesting that, in an open conformation in which this loop presumably moves away (Liu et al. 2015; Takahashi et al. 2019), the pocket would be easily accessible to many different ligands.

Second, the pocket itself is clearly amphipathic, as it is lined with apolar side chains on one side (F109, I111, M117, F121, A147, A148) and polar and charged residues on the other (R126, Y144, D146, D173 [Fig. 4B]). Our analysis shows that while the hydrophobic side of the pocket can form favorable interactions with aliphatic side chains of L-alanine, L-valine, Lleucine, L-isoleucine, and L-proline, the hydrophilic side serves to anchor the amino and carboxyl groups of all amino acid ligands of CtaA LBD and, in addition, provides a hydrogenbonding acceptor $\left(\mathrm{D} 146 \mathrm{O}^{\delta 2}\right)$ for the side-chain oxygen of an amino acid ligand with a small polar side chain, L-serine.

Third, comparisons of the ligand-binding pockets of CtaA LBD bound to different amino acids (Fig. 6A to F) reveal that the solvent-accessible volume of the pocket (calculated using a probe radius of $1.4 \AA$ ) (Dundas et al. 2006) increases from a value of $133 \AA^{3}$ for the CtaA LBD/L-alanine complex to $270 \AA^{3}$ for the $\mathrm{CtaA} \mathrm{LBD} / \mathrm{L}-\mathrm{leucine}$ complex. As illustrated in Figure $6 \mathrm{G}$, the pocket volume follows the order of the size of the ligand, demonstrating high structural plasticity of the ligand-binding site.

Superimposition of the structures of all CtaA LBD/amino acid complexes revealed that variation of the volume of the ligand-binding pocket in CtaA LBD is achieved without major changes in the protein fold. Calculation of the residue-byresidue $C_{\alpha}$ atom RMSD to the mean structure showed minor differences in the backbone conformation of loops $\beta 1 \beta 2, \beta 2 \alpha 3$, $\alpha 3 \beta 3, \beta 4 \beta 5$, and helix $\alpha 3$ (Fig. $6 \mathrm{H}$, Supplementary Table S1). In addition, analysis of the superimposed structures provided evidence of conformational variability of the protein side chains in the ligand-binding pocket, which contributes to the ability of the receptor to accommodate structurally diverse ligands. As illustrated in Figures 5A and B and 6I, the side chains of one or both I111 and F109 in the structures of the complexes with larger amino acids (L-leucine and L-isoleucine) adopt conformations different from those seen in the complexes with smaller amino acids (e.g., L-serine, L-valine), which creates a larger hydrophobic pocket into which the long, aliphatic side chain of the ligand can fit. Thus, our analysis demonstrates that the broad specificity of the amino acid chemoreceptor CtaA is afforded by the main chain and side chain plasticity of its amphipathic ligand-binding pocket.

\section{DISCUSSION}

Bacteria use amino acids as nutrients or environmental cues, and free amino acids often elicit a strong attractant response in motile bacteria. Amino acids released by plant roots, for example, have been shown to produce positive chemotactic responses in bacteria present in the rhizosphere (Gaworzewska and Carlile, 1982; Oku et al. 2012). Directed migration of bacteria toward plant roots is required for initiation of direct plant-bacteria interactions and colonization of the roots themselves. Indeed, nonmotile or nonchemotactic mutants of $P$. fluorescens, a PGPR, showed a significantly reduced ability to colonize tomato and potato roots in wild-type competition experiments (Dekkers et al. 1998; de Weert et al. 2002; De Weger et al. 1987; Simons et al. 1996). In particular, directed movement to amino acids released by plant roots has been shown to play an important role in root colonization by P. fluorescens, a PGPR (Oku et al. 2012). P. fluorescens Pf0-1 is attracted to all 20 natural L-amino acids. This response is mediated by three major chemosensory proteins for amino acids (CtaA, $\mathrm{CtaB}$, and $\mathrm{CtaC}$ ). CtaA, which, as we have shown in this study, contains a dCache LBD, mediates chemotaxis toward all amino acids except for glutamine and glutamic acid (Oku et al. 2012).

Amino acid chemoreceptors with dCache LBDs are widespread; putative receptors of this type have been found not only in each subgroup of Proteobacteria but also phyla Firmicutes, Thermotogae, Spirochaetes, and even in archaea (Liu et al. 2015). A small but growing number of reports on ligandbinding profiles of characterized receptors from this structural family provided evidence that promiscuous, broad-specificity receptors that sense more than one amino acid are common in environmental and pathogenic bacteria (Glekas et al. 2012; Nishiyama et al. 2016; Oku et al. 2012; Rico-Jiménez et al. 2013; Taguchi et al. 1997; Takahashi et al. 2019; Webb et al. 2017). Our analysis of the structural basis of amino acid recognition by $P$. fluorescens chemoreceptor CtaA provided a rationale for how dCache-type receptors can recognize a range of chemically distinct amino acids.

Our study focused on the seven amino acid ligands of CtaA that directly bind to its LBD; the remaining 11 amino acids that signal through $\mathrm{CtaA}$ are thought to activate the receptor via yet unknown indirect mechanisms. The dissociation constants for amino acid binding to CtaA LBD range from approximately $5 \mu \mathrm{M}$ for L-valine and L-alanine to approximately $450 \mu \mathrm{M}$ for $\mathrm{L}$-arginine, which falls within the range of values reported for amino acid ligand binding to other dCache LBDs (e.g., $86 \mu \mathrm{M}$

Table 2. Stabilizing interactions between ligands and CtaA ligand-binding domain (LBD) ${ }^{\mathrm{a}}$

\begin{tabular}{|c|c|c|}
\hline Ligand moiety & Protein moiety & Type of interaction \\
\hline Amino group & Y1440 ${ }^{\eta}$ (except L-proline), D1460 $\mathrm{O}^{\delta 1}, \mathrm{D} 173 \mathrm{O}^{\delta 2}$ & H-bond \\
\hline Carboxyl group oxygen & $\mathrm{R} 126 \mathrm{~N}^{\varepsilon}, \mathrm{R} 126 \mathrm{~N}^{\eta 2}, \mathrm{~W} 128 \mathrm{~N}^{\varepsilon 1}$, main-chain amino group of $\mathrm{A} 147$ & H-bond \\
\hline \multicolumn{3}{|c|}{ 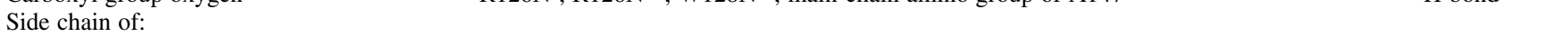 } \\
\hline L-valine & Y101, F109, I111, M117, F121, W128, A148 & VDW \\
\hline L-alanine & Y101, F109, I111, M117 & VDW \\
\hline L-serine $\left(\mathrm{O}^{\gamma}\right)$ & $\mathrm{D} 146 \mathrm{O}^{\delta 2}$ & H-bond \\
\hline L-serine & F109, Y101, I111, M117, F121, A148 & VDW \\
\hline L-leucine & Y101, I111, M117, F121, A147, A148 & VDW \\
\hline L-isoleucine & A99, Y101, F109, I111, M117, F121, A147, A148 & VDW \\
\hline L-proline & Y101, F109, I111, M117, F121, W128 & VDW \\
\hline
\end{tabular}

\footnotetext{
${ }^{\mathrm{a}} \mathrm{H}$-bond = hydrogen bond, VDW = van der Waals contact.
} 
for CjTlp3 LBD [Liu et al. 2015], 4.7-213 $\mu \mathrm{M}$ for Mlp24 LBD [Takahashi et al. 2019], 1 to $1,000 \mu \mathrm{M}$ for B. subtilis McpC LBD [Glekas et al. 2012]). The structural analysis of CtaA LBD revealed two putative ligand-binding pockets, one in the membrane-distal and the other in the membrane-proximal domains. Somewhat unexpectedly, we found that both polar and nonpolar amino acids bind to the same pocket in the membrane-distal subdomain and in a very similar orientation. Analysis of the crystal structures of the CtaA LBD complexes with amino acids identified the factors that enable the receptor
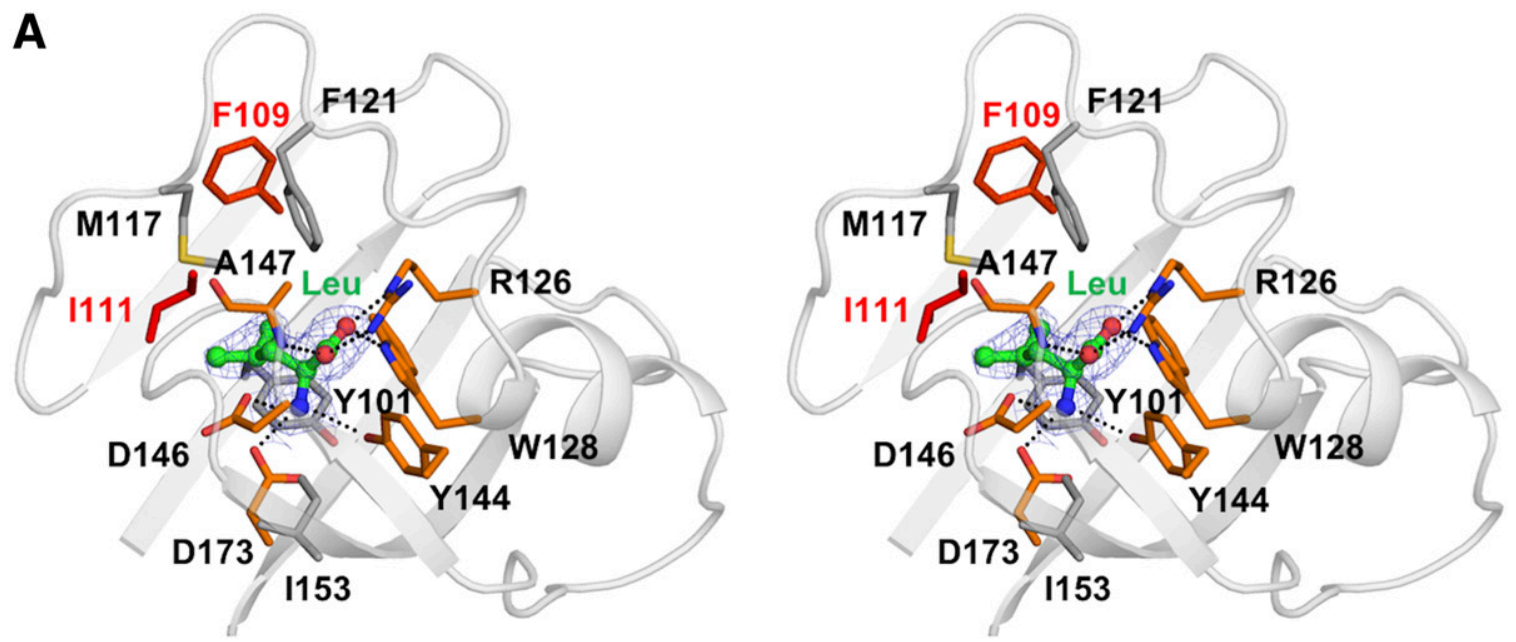

B
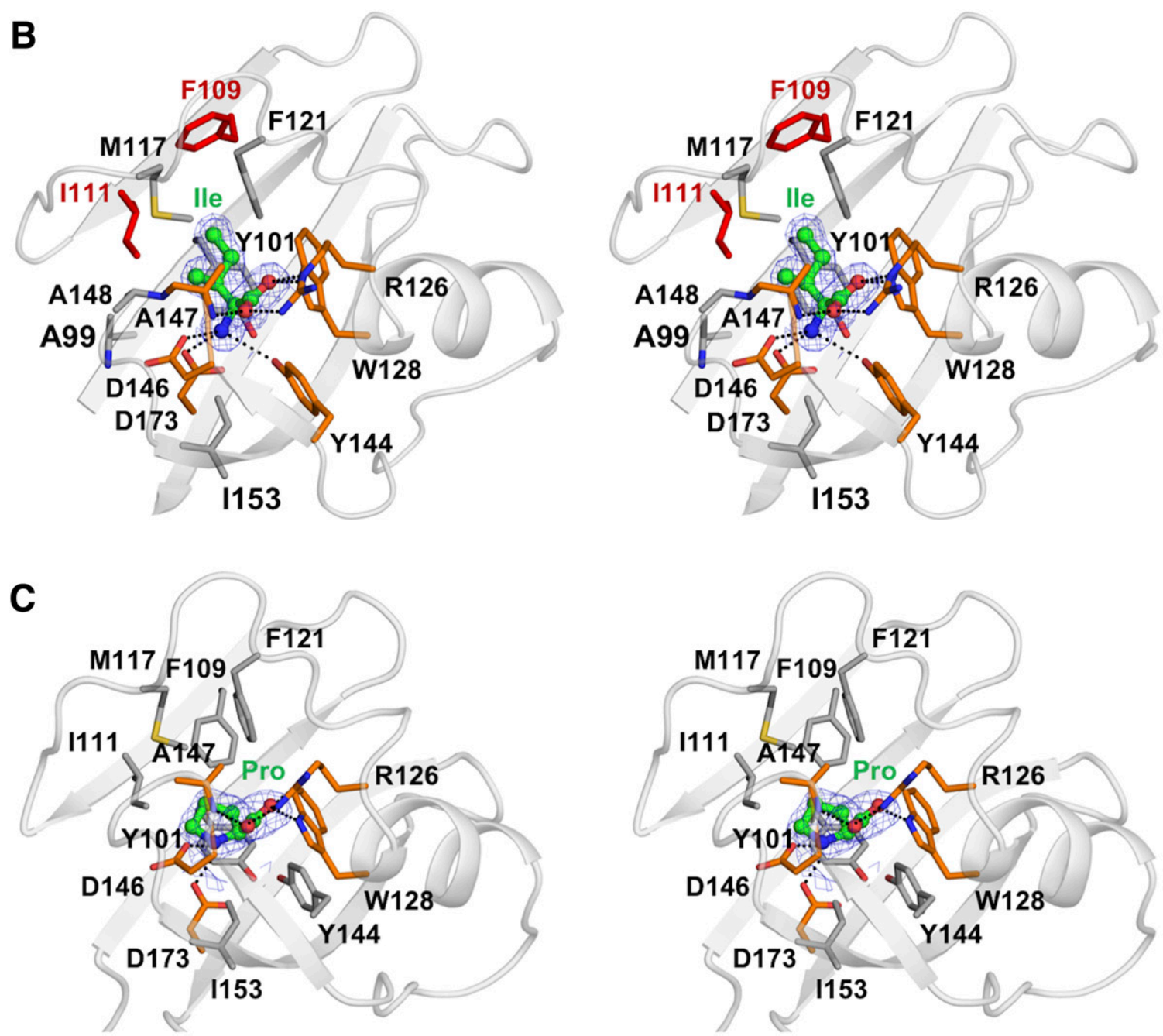

Fig. 5. Stereo diagrams showing simulated annealing omit electron density for amino acids bound to the membrane-distal subdomain of Pseudomonas fluorescens $\mathrm{CtaA}$ in respective complexes with $\mathbf{A}, \mathrm{L}-$ leucine, $\mathbf{B}, \mathrm{L}$-isoleucine, and $\mathbf{C}, \mathrm{L}-$ proline. The ligands are shown in all-atom ball-and-stick representation with $\mathrm{C}$ atoms colored green. The map was contoured at the $1.0 \sigma$ level. The residues that form hydrogen bonds and van der Waals contacts with the ligand are shown as sticks with carbons colored orange and gray, respectively. The side chains of I111 and/or F109 in the complexes with L-leucine and L-isoleucine are colored red to allow comparison of their rotameric conformations with those seen in other complexes. 
A

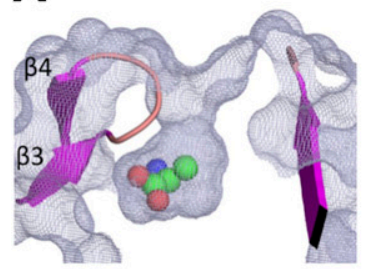

L-alanine

E

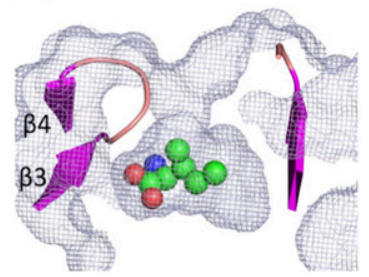

L-isoleucine
B

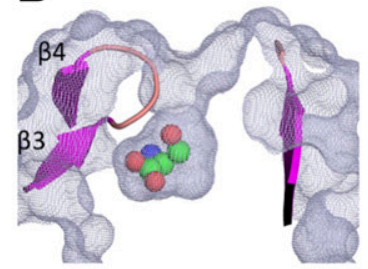

L-serine

F

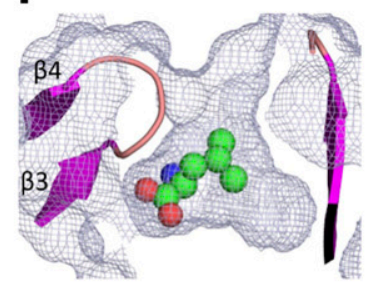

L-leucine
C

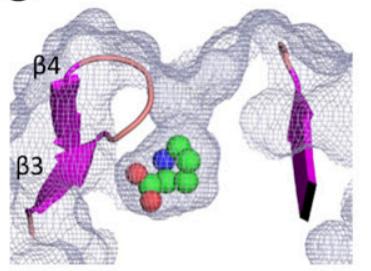

L-proline

G

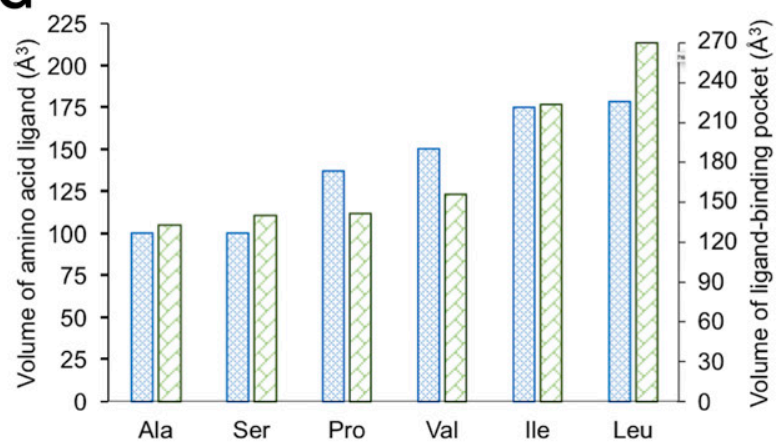

volume of ligand-binding pocket in CtaA-LBD volume of amino acid ligand

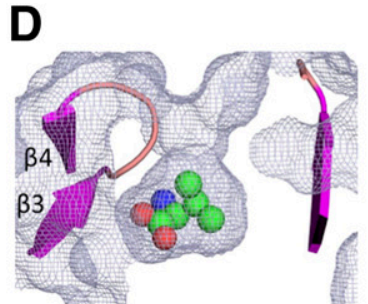

L-valine
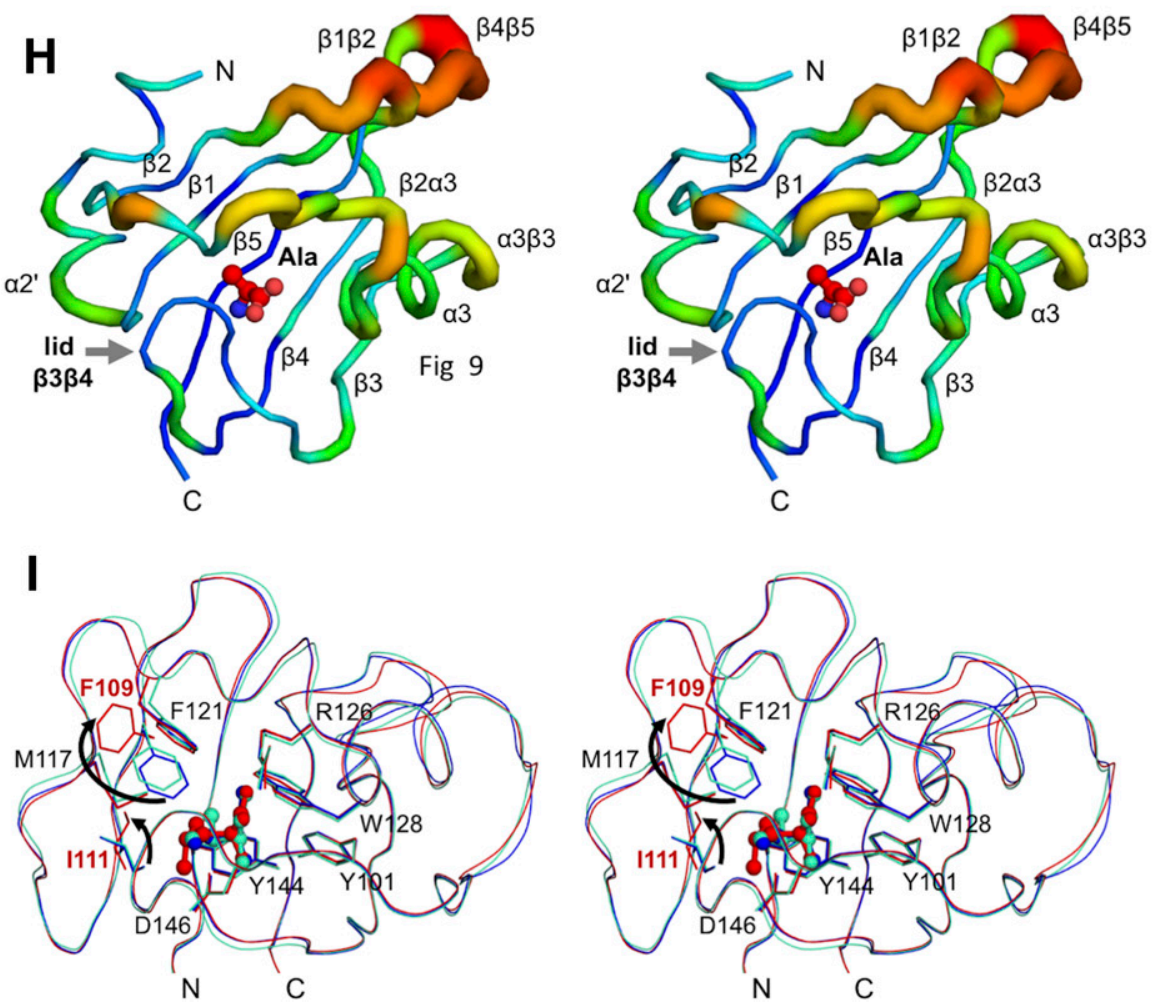

Fig. 6. Analysis of the ligand-binding pocket in the CtaA ligand-binding domain (LBD). Cross-sections across the protein molecule in the CtaA LBD complexes with A, L-alanine, B, L-serine, $\mathbf{C}$, L-proline, D, L-valine, $\mathbf{E}$, L-isoleucine, and $\mathbf{F}$, L-leucine showing the ligand-binding pocket in similar orientations. The ligand molecules are shown in ball representation and colored according to atom type, with carbon atoms in green, nitrogen in blue, and oxygen in red. The protein moiety is shown in cartoon representation. G, Changes in the probe-accessible volume of the ligand-binding pocket in CtaA LBD induced by the binding of amino acids (for calculations, ligand atoms have been excluded from the model). For the L-isoleucine complex, the average value for the two subunits in the asymmetric unit is shown. The values of the volumes of amino acids in solution are from Harpaz et al. (1994). H, Stereo diagram showing residues 85 to 175 of the CtaA LBD/L-alanine complex, drawn with the protein backbone radius proportional to the residue-by-residue $\mathrm{C}_{\alpha}$ atom root-meansquare deviation (RMSD) to the mean structure for the superimposition of the total of eight subunits in the asymmetric units of all form A and form B crystals. Structure superimposition and calculation of RMSD values were performed using the GESAMT algorithm from the CCP4 suite (Winn et al. 2011) and the figure was prepared using PyMol (Schrödinger, 2016). The color gradient runs from blue (the smallest RMSD) to red (the largest RMSD). I, Stereo diagram highlighting differences between the superimposed structures of the CtaA LBD/L-serine (blue), CtaA LBD/L-leucine (red), and CtaA LBD/L-valine (cyan) complexes in the ligand-binding region. The black arrows illustrate how alternative rotamers of I111 and F109 in the complex with L-leucine create extra space required to accommodate the ligand's long, aliphatic side chain. 
to recognize many chemically different amino acids, which are outlined and discussed below.

Our analysis suggests that the most significant determinant of CtaA specificity as a receptor for amino acids is the protein side chains that recognize the amino and carboxyl groups-the invariant part - of the amino acid ligand (R126, W128, Y144, D146, and D173). Comparison of the sequence of CtaA with VcMlp37 and CjTlp3 highlights the strong conservation of these residues in other amino acid receptors containing a dCache LBD (Supplementary Fig. S2). Previous mutagenesis studies provided evidence that these residues play an important role in ligand recognition. Alanine substitutions of the CjTlp3 LBD residues corresponding to W128, Y144, D146, and D173 in CtaA drastically reduced the affinity of isoleucine binding (Liu et al. 2015). Similarly, alanine substitutions of the $P$. aeruginosa PctA LBD residues equivalent to R126, W128, Y144, D146, and D173 in CtaA significantly reduced or abolished its interactions with amino acids (Rico-Jiménez et al. 2013). Furthermore, when the corresponding VcMlp37 variants were expressed in the $V$. cholerae mutant strain mlp24/mlp37, they showed defects in mediating chemotaxis toward amino acids (Nishiyama et al. 2016). Cumulatively, these observations indicate that dCache-type receptors share a common mechanism of recognition of amino acid ligands via their invariant moiety, the amino and carboxyl groups. This notion is supported by the observation that $\mathrm{L}$-isoleucine, for example, binds to LBDs of CtaA and CjTlp3 in a remarkably similar mode
(Supplementary Fig. S1), despite the very low sequence identity $(<17 \%)$ between the two domains.

Our analysis of the CtaA LBD/amino acid complexes reveals that the ability of $\mathrm{CtaA}$ to recognize a range of structurally and chemically distinct amino acids, including ones with aliphatic (L-leucine), small polar (L-serine), and large charged (L-arginine) side chains is afforded by its easily accessible, plastic, amphipathic pocket, which can expand or contract according to the size of the ligand side chain. Ligand size-dependent variation of the pocket volume implies that structural plasticity of the LBD is essential for CtaA to carry out its physiological role.

A comparison of the L-leucine- and L-alanine-bound structures shows that almost a twofold increase in the volume of the ligand-binding pocket is achieved via two protein side chains (F109 and I111) adopting alternative conformations and via minor adjustments in the conformation of the main chain. Remarkably, the observed ligand-dependent variations in the volume of the binding pocket do not affect the position of the loop $\beta 3 \beta 4$ that functions as a lid over the ligand-binding pocket (Fig. 6H). This is an important and counter-intuitive result, as previous studies of the free and ligand-bound forms of dCache LBDs identified this loop as highly mobile (Liu et al. 2015; Takahashi et al. 2019). Given that this loop is one of the most conserved elements in the LBD, as its amino acid sequence forms part of the Cache motif (Anantharaman and Aravind 2000; Upadhyay et al. 2016), this observation strongly suggests that the conserved closed conformation of this loop adopted

Table 3. Data collection and processing statistics

\begin{tabular}{|c|c|c|c|c|c|c|c|}
\hline \multirow[b]{2}{*}{ Dataset } & \multirow{2}{*}{$\begin{array}{c}\text { CtaA LBD } \\
\text { native }^{a}\end{array}$} & \multicolumn{6}{|c|}{ CtaA LBD in complex with } \\
\hline & & L-alanine & L-serine & L-isoleucine & L-leucine & L-valine & L-proline \\
\hline $\begin{array}{l}\text { Space group } \\
a, b, c(\AA)\end{array}$ & $\begin{array}{c}I 2_{1} 2_{1} 2_{1} \\
66.1,75.5,113.7\end{array}$ & $\begin{array}{c}I 2{ }_{1} 2_{1} 2_{1} \\
66.3,75.3,113.7\end{array}$ & $\begin{array}{c}I 2_{1} 2_{1} 2_{1} \\
67.2,76.0,113.3\end{array}$ & $\begin{array}{c}C 2 \\
133.5,70.7,71.0\end{array}$ & $\begin{array}{c}I 2_{1} 2_{1} 2_{1} \\
70.2,71.4,112.8\end{array}$ & $\begin{array}{c}I 2_{1} 2_{1} 2_{1} \\
60.2,71.8,112.3\end{array}$ & $\begin{array}{c}I 2_{1} 2_{1} 2_{1} \\
61.7,71.7,112.3\end{array}$ \\
\hline $\begin{array}{l}\text { Resolution } \\
\text { range }(\AA)\end{array}$ & $\begin{array}{c}37.5-1.95 \\
(2.06-1.95)\end{array}$ & $\begin{array}{c}37.6-2.0 \\
(2.05-2.00)\end{array}$ & $\begin{array}{c}33.8-1.9 \\
(1.94-1.90)\end{array}$ & $\begin{array}{c}40.0-1.9 \\
(2.00-1.90)\end{array}$ & $\begin{array}{c}35.0-2.0 \\
(2.11-2.00)\end{array}$ & $\begin{array}{c}40.0-2.1 \\
(2.22-2.10)\end{array}$ & $\begin{array}{c}40.0-2.0 \\
(2.11-2.00)\end{array}$ \\
\hline $\begin{array}{l}\text { Total number of } \\
\text { reflections }\end{array}$ & $\begin{array}{l}142,232 \\
(21,483)\end{array}$ & $\begin{array}{l}63,624 \\
(4,831)\end{array}$ & $\begin{array}{l}79,149 \\
(5,083)\end{array}$ & $\begin{array}{l}16,4140 \\
(23,850)\end{array}$ & $\begin{array}{l}131,407 \\
(19,591)\end{array}$ & $\begin{array}{l}102,547 \\
(15,050)\end{array}$ & $\begin{array}{l}118,988 \\
(17,409)\end{array}$ \\
\hline $\begin{array}{l}\text { Number of unique } \\
\text { reflections }\end{array}$ & $\begin{array}{l}20,853 \\
(3,032)\end{array}$ & $\begin{array}{l}16,753 \\
(1,259)\end{array}$ & $\begin{array}{l}21,256 \\
(1,353)\end{array}$ & $\begin{array}{l}42,486 \\
(6,131)\end{array}$ & $\begin{array}{l}19,503 \\
(2,817)\end{array}$ & $\begin{array}{l}145,999 \\
(2,108)\end{array}$ & $\begin{array}{l}17,230 \\
(2,471)\end{array}$ \\
\hline Completeness (\%) & $99(100)$ & $87(89)$ & $92(94)$ & $97(96)$ & $100(100)$ & $100(100)$ & $100(100)$ \\
\hline Redundancy & $5.1(5.3)$ & $3.8(3.8)$ & $3.7(3.8)$ & $3.9(3.9)$ & $6.7(7.0)$ & $7.0(7.1)$ & $6.9(7.0)$ \\
\hline Mean $I / \sigma(I)$ & $16.2(4.8)$ & $12.0(3.2)$ & $12.7(2.5)$ & $16.5(4.4)$ & $13.7(4.8)$ & $17.6(4.8)$ & $11.7(4.4)$ \\
\hline$R_{\text {merge }}$ & $0.07(0.39)$ & $0.05(0.30)$ & $0.05(0.35)$ & $0.05(0.31)$ & $0.08(0.31)$ & $0.07(0.43)$ & $0.09(0.38)$ \\
\hline
\end{tabular}

a Values in parentheses are for the highest resolution shell. LBD = ligand-binding domain.

Table 4. Refinement statistics ${ }^{\mathrm{a}}$

\begin{tabular}{|c|c|c|c|c|c|c|c|}
\hline \multirow[b]{2}{*}{ Dataset } & \multirow[b]{2}{*}{ CtaA LBD native } & \multicolumn{6}{|c|}{ CtaA LBD in complex with } \\
\hline & & L-alanine & L-serine & L-isoleucine & L-leucine & L-valine & L-proline \\
\hline Resolution (§) & $31.5-2.0$ & $31.4-2.0$ & $33.8-1.9$ & $37.4-1.9$ & $33.3-2.0$ & $35.7-2.2$ & $36.0-2.0$ \\
\hline Residues/atoms/waters & $229 / 1,764 / 115$ & $226 / 1,778 / 149$ & $228 / 1,800 / 158$ & $3,793 / 476 / 336$ & $232 / 1,820 / 128$ & $213 / 1,729 / 157$ & $222 / 1,810 / 193$ \\
\hline $\mathrm{R} / \mathrm{R}_{\text {free }}$ & $0.191 / 0.239$ & $0.204 / 0.249$ & $0.196 / 0.233$ & $0.180 / 0.225$ & $0.191 / 0.238$ & $0.202 / 0.286$ & $0.20 / 0.26$ \\
\hline \multicolumn{8}{|l|}{ Average B factors $\left(\AA^{2}\right)$} \\
\hline Protein & 41.8 & 42.3 & 38.2 & 28.4 & 41.0 & 41.4 & 38.5 \\
\hline Water & 48.0 & 43.0 & 40.3 & 31.6 & 41.3 & 40.2 & 48.2 \\
\hline Ligand & 29.6 & 27.3 & 23.4 & 17.8 & 33.0 & 28.1 & 24.4 \\
\hline \multicolumn{8}{|l|}{ RMSD from ideality } \\
\hline Bond lengths $(\AA)$ & 0.01 & 0.01 & 0.01 & 0.01 & 0.01 & 0.01 & 0.01 \\
\hline Bond angles $\left({ }^{\circ}\right)$ & 1.1 & 1.5 & 1.5 & 1.5 & 1.6 & 1.5 & 1.2 \\
\hline \multicolumn{8}{|l|}{ Ramachandran plot $(\%)$} \\
\hline Favored & 99 & 98 & 98 & 98 & 98 & 96 & 97 \\
\hline Allowed & 1 & 2 & 2 & 2 & 2 & 4 & 3 \\
\hline Outliers & 0 & 0 & 0 & 0 & 0 & 0 & 0 \\
\hline
\end{tabular}

${ }^{\mathrm{a}} \mathrm{LBD}=$ ligand-binding domain, RMSD = root-mean-square deviation. 
upon binding of various ligands forms an important part of the signaling mechanism.

Our analysis suggests that an amphipathic character of the ligand-binding pocket is another factor that enables promiscuous interactions of the receptor with both polar and nonpolar amino acids. Finally, we note that access to this pocket is through a short tunnel that remains open, even in the closed form of the protein, and that a similar opening connecting the pocket to the bulk solvent has been reported for one of the subunits in the L-serine-bound structure of a different broad-specificity receptor for amino acids, VcMlp37 (Nishiyama et al. 2016). In contrast, the only dCache chemoreceptor with narrow specificity that has, so far, been structurally characterized-CjTlp3 — completely engulfs its ligand L-isoleucine, fully shielding it from the bulk solvent. As illustrated in Figure 6A to $\mathrm{F}$, the short tunnel connects the end of the side chain of the bound amino acid and the surface of the protein molecule. Although we could not obtain the structure of the CtaA LBD complex with Larginine, modeling of L-arginine, based on the coordinates of bound L-leucine, places its side chain into the tunnel, with the aliphatic part of the side chain stabilized against the hydrophobic side of the pocket and its positively charged guanidine group exposed on the protein surface. In conclusion, our biophysical and structural analysis of CtaA LBD provided important insights into the structure and function of promiscuous dCache chemoreceptors. The findings presented here provide a foundation for future systematic genetic and biochemical studies.

\section{RCSB PDB accession numbers.}

The coordinates and structure factors of the CtaA LBD/amino acid complexes obtained by cocrystallization with L-alanine, Lvaline, L-serine, L-leucine, L-isoleucine, and L-proline have been deposited in the RCSB PDB under accession codes 6PXY, 6Q0F, 6PY5, 6PY4, 6PY3, and 6Q0G. The coordinates and structure factors of CtaA LBD that was crystallized with no exogenous amino acid added but modeled as an L-alanine complex have been deposited under PDB accession code 6PYI.

\section{MATERIALS AND METHODS}

\section{Protein expression, purification, crystallization, and data collection.}

Recombinant $P$. fluorescens Pf0-1 CtaA-LBD (residues 34 to 277 plus an additional $\mathrm{N}$-terminal GIDPFT sequence as a cloning artifact) was expressed and purified as previously described (UdDin and Roujeinikova 2016). Prior to crystallization, CtaA-LBD was concentrated to $8 \mathrm{mg} / \mathrm{ml}$. Form A crystals of CtaA-LBD were obtained by the hanging-drop vapor-diffusion method using a reservoir solution consisting of $2.0 \mathrm{M}$ ammonium sulfate and $0.1 \mathrm{M}$ Tris- $\mathrm{HCl}, \mathrm{pH}$ 8.0. The crystals had a symmetry of space group $I 2{ }_{1} 22_{1} 2_{1}$ (form A) with unit-cell dimensions $a=66.1 \AA$, $b=$ $75.5 \AA, c=113.7 \AA$ and had one protein molecule in the asymmetric unit. Cocrystallization with $5 \mathrm{mM} \mathrm{L}$-alanine, Lvaline, L-serine, L-leucine, or L-proline under similar conditions produced form A crystals of the respective complexes with similar unit-cell parameters (Table 3). Cocrystallization with $5 \mathrm{mM}$ L-isoleucine under similar conditions produced form $\mathrm{B}$ crystals that belonged to space group $C 2$ with unit-cell parameters $a=133.5 \AA, b=70.7 \AA, c=71.0 \AA$, and $\beta=122$ degrees and had two protein subunits in the asymmetric unit. X-ray diffraction data were collected at 100 kelvins, using the MX1 and MX2 beamlines of the Australian Synchrotron. All diffraction data were processed and scaled using iMOSFLM (Battye et al. 2011) and AIMLESS (Evans and Murshudov 2013) from the CCP4 software suite (Winn et al. 2011). Data collection and processing statistics are summarized in Table 3.

\section{Structure determination and analysis.}

The crystal structure of the CtaA-LBD was solved by molecular replacement with Phaser (McCoy et al. 2007), using the diffraction data for the form A cocrystals with L-alanine and the structure of Mlp37 from V. cholerae (PDB ID 3C8C [Nishiyama et al. 2016]) as a search model. The model from the molecular replacement solution was rebuilt to completion through iterative cycles of manual adjustment in Coot (Emsley et al. 2010) and refinement using PHENIX (Adams et al. 2010). The refined coordinates of the protein subunit in form $A$ crystals of the CtaA-LBD/Ala complex were used for phasing the form $\mathrm{A}$ and form $\mathrm{B}$ data for the CtaA-LBD, crystallized with or without amino acids, by molecular replacement. Analysis of the stereochemical quality of the models was performed using MolProbity (Chen et al. 2010). The refinement statistics are summarized in Table 4. The structure and sequence alignment figures were prepared using PyMol (Schrödinger 2016) and ESPript (Robert and Gouet 2014), respectively. The accessible surface area was calculated using AREAIMOL from CCP4.

\section{ITC experiments.}

P. fluorescens CtaA-LBD was dialyzed against a buffer containing $10 \mathrm{mM}$ Tris, $\mathrm{pH} 8.0$ and $200 \mathrm{mM} \mathrm{NaCl}$. Ligand solutions were prepared by dissolving them in the dialysis buffer, at a concentration of $0.5 \mathrm{mM}$ for L-valine, L-alanine, $\mathrm{L}$-serine, and L-proline, $1 \mathrm{mM}$ for L-leucine, $3 \mathrm{mM}$ for $\mathrm{L}$ isoleucine, and $10 \mathrm{mM}$ for all other natural amino acids. Measurements were performed at $25^{\circ} \mathrm{C}$ using a VP-ITC MicroCal calorimeter (Malvern Instruments). The protein sample $(10 \mu \mathrm{M})$ in a $1.45-\mathrm{ml}$ reaction cell was injected with 25 successive 10- $\mu$ l aliquots of the ligand solution at a spacing of $300 \mathrm{~s}$. Binding isotherms were produced by plotting the heat change per injection against the molar ratio of a ligand to CtaA-LBD. The data were fitted with Origin 7 (OriginLab) to a single-site binding model using nonlinear least-squares regression.

\section{ACKNOWLEDGMENTS}

The authors thank D. Maksel and G. K. W. Kong at the Monash Macromolecular Crystallization Facility for their assistance in setting up robotic crystallization screens. Part of this research was undertaken on the MX1 and MX2 beamlines of the Australian Synchrotron, Victoria, Australia. We thank the Australian Synchrotron staff for their help with data collection.

\section{AUTHOR-RECOMMENDED INTERNET RESOURCE}

European Bioinformatics Institute PDBePISA server: https://www.ebi.ac.uk/pdbe/pisa

\section{LITERATURE CITED}

Adams, P. D., Afonine, P. V., Bunkóczi, G., Chen, V. B., Davis, I. W., Echols, N., Headd, J. J., Hung, L.-W., Kapral, G. J., Grosse-Kunstleve, R. W., McCoy, A. J., Moriarty, N. W., Oeffner, R., Read, R. J., Richardson, D. C., Richardson, J. S., Terwilliger, T. C., and Zwart, P. H. 2010. PHENIX: A comprehensive Python-based system for macromolecular structure solution. Acta Crystallogr. D Biol. Crystallogr. 66:213-221.

Anantharaman, V., and Aravind, L. 2000. Cache-A signaling domain common to animal $\mathrm{Ca}^{2+}$-channel subunits and a class of prokaryotic chemotaxis receptors. Trends Biochem. Sci. 25:535-537.

Battye, T. G. G., Kontogiannis, L., Johnson, O., Powell, H. R., and Leslie, A. G. 2011. iMOSFLM: A new graphical interface for diffraction-image processing with MOSFLM. Acta Crystallogr. D Biol. Crystallogr. 67: 271-281.

Berman, H., Henrick, K., Nakamura, H., and Markley, J. L. 2007. The worldwide Protein Data Bank (wwPDB): Ensuring a single, uniform archive of PDB data. Nucleic Acids Res. 35 (Database):D301-D303.

Briegel, A., Ortega, D. R., Tocheva, E. I., Wuichet, K., Li, Z., Chen, S., Müller, A., Iancu, C. V., Murphy, G. E., Dobro, M. J., Zhulin, I. B., and 
Jensen, G. J. 2009. Universal architecture of bacterial chemoreceptor arrays. Proc. Natl. Acad. Sci. U.S.A. 106:17181-17186.

Chen, V. B., Arendall, W. B., 3rd, Headd, J. J., Keedy, D. A., Immormino, R. M., Kapral, G. J., Murray, L. W., Richardson, J. S., and Richardson, D. C. 2010. MolProbity: All-atom structure validation for macromolecular crystallography. Acta Crystallogr. D Biol. Crystallogr. 66:12-21.

Compant, S., Duffy, B., Nowak, J., Clément, C., and Barka, E. A. 2005. Use of plant growth-promoting bacteria for biocontrol of plant diseases: Principles, mechanisms of action, and future prospects. Appl. Environ. Microbiol. 71:4951-4959.

de Weert, S., Vermeiren, H., Mulders, I. H., Kuiper, I., Hendrickx, N., Bloemberg, G. V., Vanderleyden, J., De Mot, R., and Lugtenberg, B. J. 2002. Flagella-driven chemotaxis towards exudate components is an important trait for tomato root colonization by Pseudomonas fluorescens. Mol. Plant-Microbe Interact. 15:1173-1180.

De Weger, L. A., van der Vlugt, C. I., Wijfjes, A. H., Bakker, P. A., Schippers, B., and Lugtenberg, B. 1987. Flagella of a plant-growthstimulating Pseudomonas fluorescens strain are required for colonization of potato roots. J. Bacteriol. 169:2769-2773.

Dekkers, L. C., van der Bij, A. J., Mulders, I. H. M., Phoelich, C. C., Wentwoord, R. A., Glandorf, D. C., Wijffelman, C. A., and Lugtenberg, B. J. J. 1998. Role of the O-antigen of lipopolysaccharide, and possible roles of growth rate and of NADH:ubiquinone oxidoreductase (nuo) in competitive tomato root-tip colonization by Pseudomonas fluorescens WCS365. Mol. Plant-Microbe Interact. 11:763-771.

Dundas, J., Ouyang, Z., Tseng, J., Binkowski, A., Turpaz, Y., and Liang, J. 2006. CASTp: Computed atlas of surface topography of proteins with structural and topographical mapping of functionally annotated residues. Nucleic Acids Res. 34 (Web Server):W116-W118.

Emsley, P., Lohkamp, B., Scott, W. G., and Cowtan, K. 2010. Features and development of Coot. Acta Crystallogr. D Biol. Crystallogr. 66:486-501.

Evans, P. R., and Murshudov, G. N. 2013. How good are my data and what is the resolution? Acta Crystallogr. D Biol. Crystallogr. 69:1204-1214.

Finn, R. D., Bateman, A., Clements, J., Coggill, P., Eberhardt, R. Y., Eddy, S. R., Heger, A., Hetherington, K., Holm, L., Mistry, J., Sonnhammer, E. L., Tate, J., and Punta, M. 2014. Pfam: The protein families database. Nucleic Acids Res. 42 (D1):D222-D230.

Gaworzewska, E. T., and Carlile, M. J. 1982. Positive chemotaxis of Rhizobium leguminosarum and other bacteria towards root exudates from legumes and other plants. J. Gen. Microbiol. 128:1179-1188.

Glekas, G. D., Mulhern, B. J., Kroc, A., Duelfer, K. A., Lei, V., Rao, C. V., and Ordal, G. W. 2012. The Bacillus subtilis chemoreceptor McpC senses multiple ligands using two discrete mechanisms. J. Biol. Chem. 287:39412-39418.

Glick, B. R. 2012. Plant growth-promoting bacteria: Mechanisms and applications. Scientifica (Cairo) 2012:963401.

Harpaz, Y., Gerstein, M., and Chothia, C. 1994. Volume changes on protein folding. Structure 2:641-649.

Krell, T., Lacal, J., Muñoz-Martínez, F., Reyes-Darias, J. A., Cadirci, B. H., García-Fontana, C., and Ramos, J. L. 2011. Diversity at its best: Bacterial taxis. Environ. Microbiol. 13:1115-1124.

Krissinel, E., and Henrick, K. 2004. Secondary-structure matching (SSM), a new tool for fast protein structure alignment in three dimensions. Acta Crystallogr. D Biol. Crystallogr. 60:2256-2268.

Krissinel, E., and Henrick, K. 2007. Inference of macromolecular assemblies from crystalline state. J. Mol. Biol. 372:774-797.

Lacal, J., García-Fontana, C., Muñoz-Martínez, F., Ramos, J. L., and Krell, T. 2010. Sensing of environmental signals: Classification of chemoreceptors according to the size of their ligand binding regions. Environ. Microbiol. 12:2873-2884.

Liu, Y. C., Machuca, M. A., Beckham, S. A., Gunzburg, M. J., and Roujeinikova, A. 2015. Structural basis for amino-acid recognition and transmembrane signalling by tandem Per-Arnt-Sim (tandem PAS) chemoreceptor sensory domains. Acta Crystallogr. D Biol. Crystallogr. 71:2127-2136.

Lugtenberg, B., and Kamilova, F. 2009. Plant-growth-promoting rhizobacteria. Annu. Rev. Microbiol. 63:541-556.

Machuca, M. A., Johnson, K. S., Liu, Y. C., Steer, D. L., Ottemann, K. M., and Roujeinikova, A. 2017. Helicobacter pylori chemoreceptor TlpC mediates chemotaxis to lactate. Sci. Rep. 7:14089.

Machuca, M. A., Liu, Y. C., Beckham, S. A., Gunzburg, M. J., and Roujeinikova, A. 2016. The crystal structure of the tandem-PAS sensing domain of Campylobacter jejuni chemoreceptor Tlp1 suggests indirect mechanism of ligand recognition. J. Struct. Biol. 194:205-213.

Matilla, M. A., and Krell, T. 2017. Chemoreceptor-based signal sensing. Curr. Opin. Biotechnol. 45:8-14.

McCoy, A. J., Grosse-Kunstleve, R. W., Adams, P. D., Winn, M. D., Storoni, L. C., and Read, R. J. 2007. Phaser crystallographic software. J. Appl. Cryst. 40:658-674.
Nishiyama, S., Takahashi, Y., Yamamoto, K., Suzuki, D., Itoh, Y., Sumita, K., Uchida, Y., Homma, M., Imada, K., and Kawagishi, I. 2016. Identification of a Vibrio cholerae chemoreceptor that senses taurine and amino acids as attractants. Sci. Rep. 6:20866.

Oku, S., Komatsu, A., Nakashimada, Y., Tajima, T., and Kato, J. 2014. Identification of Pseudomonas fluorescens chemotaxis sensory proteins for malate, succinate, and fumarate, and their involvement in root colonization. Microbes Environ. 29:413-419.

Oku, S., Komatsu, A., Tajima, T., Nakashimada, Y., and Kato, J. 2012. Identification of chemotaxis sensory proteins for amino acids in Pseudomonas fluorescens Pf0-1 and their involvement in chemotaxis to tomato root exudate and root colonization. Microbes Environ. 27:462-469.

Ortega, Á., and Krell, T. 2014. The HBM domain: Introducing bimodularity to bacterial sensing. Protein Sci. 23:332-336.

Rico-Jiménez, M., Muñoz-Martínez, F., García-Fontana, C., Fernandez, M., Morel, B., Ortega, A., Ramos, J. L., and Krell, T. 2013. Paralogous chemoreceptors mediate chemotaxis towards protein amino acids and the non-protein amino acid gamma-aminobutyrate (GABA). Mol. Microbiol. 88:1230-1243.

Robert, X., and Gouet, P. 2014. Deciphering key features in protein structures with the new ENDscript server. Nucleic Acids Res. 42 (W1) W320-W324.

Schrödinger. 2016. The PyMOL Molecular Graphics System, Version 1.2r3pre. Schrödinger, LLC, New York.

Simons, M., van der Bij, A. J., Brand, I., de Weger, L. A., Wijffelman, C. A., and Lugtenberg, B. J. 1996. Gnotobiotic system for studying rhizosphere colonization by plant growth-promoting Pseudomonas bacteria. Mol. Plant-Microbe Interact. 9:600-607.

Taguchi, K., Fukutomi, H., Kuroda, A., Kato, J., and Ohtake, H. 1997. Genetic identification of chemotactic transducers for amino acids in Pseudomonas aeruginosa. Microbiology 143:3223-3229.

Takahashi, Y., Nishiyama, S. I., Sumita, K., Kawagishi, I., and Imada, K. 2019. Calcium ions modulate amino acid sensing of the chemoreceptor Mlp24 of Vibrio cholerae. J. Bacteriol. 201:e0779-18.

Tan, K. P., Nguyen, T. B., Patel, S., Varadarajan, R., and Madhusudhan, M. S. 2013. Depth: A web server to compute depth, cavity sizes, detect potential small-molecule ligand-binding cavities and predict the $\mathrm{pKa}$ of ionizable residues in proteins. Nucleic Acids Res. 41 (W1): W314-W321.

Ud-Din, A. I. M. S., and Roujeinikova, A. 2016. Cloning, purification, crystallization and X-ray crystallographic analysis of the periplasmic sensing domain of Pseudomonas fluorescens chemotactic transducer of amino acids type A (CtaA). Biosci. Trends 10:320-324.

Ud-Din, A. I. M. S., and Roujeinikova, A. 2017. Methyl-accepting chemotaxis proteins: A core sensing element in prokaryotes and archaea. Cell. Mol. Life Sci. 74:3293-3303.

Upadhyay, A. A., Fleetwood, A. D., Adebali, O., Finn, R. D., and Zhulin, I. B. 2016. Cache domains that are homologous to, but different from PAS domains comprise the largest superfamily of extracellular sensors in prokaryotes. PLOS Comput. Biol. 12:e1004862.

Vacheron, J., Desbrosses, G., Bouffaud, M. L., Touraine, B., MoënneLoccoz, Y., Muller, D., Legendre, L., Wisniewski-Dyé, F., and PrigentCombaret, C. 2013. Plant growth-promoting rhizobacteria and root system functioning. Front. Plant Sci. 4:356.

van Loon, L. C., Bakker, P. A., and Pieterse, C. M. 1998. Systemic resistance induced by rhizosphere bacteria. Annu. Rev. Phytopathol. 36:453-483.

Vejan, P., Abdullah, R., Khadiran, T., Ismail, S., and Nasrulhaq Boyce, A. 2016. Role of plant growth promoting rhizobacteria in agricultural sustainability-A review. Molecules 21:573.

Wadhams, G. H., and Armitage, J. P. 2004. Making sense of it all: Bacterial chemotaxis. Nat. Rev. Mol. Cell Biol. 5:1024-1037.

Webb, B. A., Compton, K. K., Del Campo, J. S. M., Taylor, D., Sobrado, P., and Scharf, B. E. 2017. Sinorhizobium meliloti chemotaxis to multiple amino acids is mediated by the chemoreceptor McpU. Mol. PlantMicrobe Interact. 30:770-777.

Winn, M. D., Ballard, C. C., Cowtan, K. D., Dodson, E. J., Emsley, P., Evans, P. R., Keegan, R. M., Krissinel, E. B., Leslie, A. G., McCoy, A., McNicholas, S. J., Murshudov, G. N., Pannu, N. S., Potterton, E. A., Powell, H. R., Read, R. J., Vagin, A., and Wilson, K. S. 2011. Overview of the CCP4 suite and current developments. Acta Crystallogr. D Biol. Crystallogr. 67:235-242.

Zhang, Z., and Hendrickson, W. A. 2010. Structural characterization of the predominant family of histidine kinase sensor domains. J. Mol. Biol. 400:335-353.

Zhou, Y. F., Nan, B., Nan, J., Ma, Q., Panjikar, S., Liang, Y. H., Wang, Y., and Su, X. D. 2008. C4-dicarboxylates sensing mechanism revealed by the crystal structures of DctB sensor domain. J. Mol. Biol. 383:49-61. 